\title{
\begin{tabular}{l|l} 
Mitraries & DSpace@MIT
\end{tabular}
}

\author{
MIT Open Access Articles
}

\section{ARCSECOND RESOLUTION MAPPING OF SULFUR DIOXIDE EMISSION IN THE CIRCUMSTELLAR ENVELOPE OF VY CANIS MAJORIS}

The MIT Faculty has made this article openly available. Please share how this access benefits you. Your story matters.

Citation: Fu, Roger R., Arielle Moullet, Nimesh A. Patel, John Biersteker, Kimberly L. Derose, and Kenneth $\mathrm{H}$. Young. "ARCSECOND RESOLUTION MAPPING OF SULFUR DIOXIDE EMISSION IN THE CIRCUMSTELLAR ENVELOPE OF VY CANIS MAJORIS." The Astrophysical Journal 746, no. 1 (January 23, 2012): 42. (C) 2012 The American Astronomical Society

As Published: http://dx.doi.org/10.1088/0004-637x/746/1/42

Publisher: IOP Publishing

Persistent URL: http://hdl.handle.net/1721.1/95624

Version: Final published version: final published article, as it appeared in a journal, conference proceedings, or other formally published context

Terms of Use: Article is made available in accordance with the publisher's policy and may be subject to US copyright law. Please refer to the publisher's site for terms of use. 


\title{
ARCSECOND RESOLUTION MAPPING OF SULFUR DIOXIDE EMISSION IN THE CIRCUMSTELLAR ENVELOPE OF VY CANIS MAJORIS
}

\author{
Roger R. Fu ${ }^{1,2}$, Arielle Moullet ${ }^{1}$, Nimesh A. Patel ${ }^{1}$, John Biersteker $^{1}$, Kimberly L. Derose ${ }^{1}$, And Kenneth H. Young ${ }^{1}$ \\ ${ }^{1}$ Harvard-Smithsonian Center for Astrophysics, 60 Garden Street, Cambridge, MA, USA; npatel@ cfa.harvard.edu \\ ${ }^{2}$ Department of Earth, Atmospheric, and Planetary Sciences, Massachusetts Institute of Technology, Cambridge, MA, USA \\ Received 2011 March 2; accepted 2011 November 22; published 2012 January 23
}

\begin{abstract}
We report Submillimeter Array observations of $\mathrm{SO}_{2}$ emission in the circumstellar envelope (CSE) of the red supergiant VY Canis Majoris, with an angular resolution of $\approx 1^{\prime \prime}$. $\mathrm{SO}_{2}$ emission appears in three distinct outflow regions surrounding the central continuum peak emission that is spatially unresolved. No bipolar structure is noted in the sources. A fourth source of $\mathrm{SO}_{2}$ is identified as a spherical wind centered at the systemic velocity. We estimate the $\mathrm{SO}_{2}$ column density and rotational temperature assuming local thermal equilibrium (LTE) as well as perform non-LTE radiative transfer analysis using RADEX. Column densities of $\mathrm{SO}_{2}$ are found to be $\sim 10^{16} \mathrm{~cm}^{-2}$ in the outflows and in the spherical wind. Comparison with existing maps of the two parent species $\mathrm{OH}$ and $\mathrm{SO}$ shows the $\mathrm{SO}_{2}$ distribution to be consistent with that of $\mathrm{OH}$. The abundance ratio $f_{\mathrm{SO}_{2}} / f_{\mathrm{SO}}$ is greater than unity for all radii larger than $3 \times 10^{16} \mathrm{~cm}$. $\mathrm{SO}_{2}$ is distributed in fragmented clumps compared to $\mathrm{SO}, \mathrm{PN}$, and $\mathrm{SiS}$ molecules. These observations lend support to specific models of circumstellar chemistry that predict $f_{\mathrm{SO}_{2}} / f_{\mathrm{SO}}>1$ and may suggest the role of localized effects such as shocks in the production of $\mathrm{SO}_{2}$ in the CSE.
\end{abstract}

Key words: astrochemistry - circumstellar matter - radio lines: stars - stars: individual (VY CMa) - stars:

late-type - supergiants

Online-only material: color figures

\section{INTRODUCTION}

Massive stars $\left(M_{*} \gtrsim 8 M_{\odot}\right)$, enter a red supergiant (RSG) phase during which the star experiences mass loss at rates of $\dot{M} \sim 10^{-5}-10^{-3} M_{\odot} \mathrm{yr}^{-1}$ (van Loon et al. 2005). The time variation of this mass-loss rate is not well constrained by theoretical studies (Yoon \& Cantiello 2010). As a result, the total amount of mass lost over the course of the RSG phase remains uncertain for a given initial mass (Smith et al. 2009). Observations of mass-loss events have shown them to be sporadic and spatially anisotropic (de Wit et al. 2008).

VY Canis Majoris (VY CMa) is an oxygen-rich RSG with an estimated mass of $M_{*} \approx 25 M_{\odot}$ and a mass-loss rate estimated to be $\dot{M} \sim(2-4) \times 10^{-4} M_{\odot} \mathrm{yr}^{-1}$ (Danchi et al. 1994; Smith et al. 2009). Optical images of this source show multiple discrete and asymmetric mass-loss events, ranging in age from 1700 to 157 years ago, that are distinct from the general flow of diffuse material (Humphreys et al. 2007). Detailed studies of millimeter/submillimeter molecular spectra of VY CMa have been carried out, revealing the chemical complexity in the envelope (Ziurys et al. 2007; Royer et al. 2010; Tenenbaum et al. 2010). Spatial structures in the visible and IR bands have also been obtained (Smith et al. 2001). High angular resolution Submillimeter Array (SMA) ${ }^{3}$ observations of VY CMa produced maps of the spatial distribution of $\mathrm{CO}$ and $\mathrm{SO}$ (Muller et al. 2007).

Millimeter wavelength observations have long shown evidence of $\mathrm{SO}_{2}$ in the circumstellar envelopes (CSEs) of oxygenrich RSGs (e.g., Guilloteau et al. 1986) and $\mathrm{SO}_{2}$ emission in VY CMa was first detected by Sahai \& Wannier (1992). Later infrared observations have also shown the production of $\mathrm{SO}_{2}$

\footnotetext{
3 The Submillimeter Array is a joint project between the Smithsonian Astrophysical Observatory and the Academia Sinica Institute of Astronomy and Astrophysics, and is funded by the Smithsonian Institution and the Academia Sinica.
}

within several radii of O-rich asymptotic giant branch stars (in the "inner wind"; Yamamura et al. 1999). From a theoretical perspective, the presence of $\mathrm{SO}_{2}$ in CSEs has been predicted by isotropic, non-equilibrium models of stellar chemistry (Scalo \& Slavsky 1980; Nejad \& Millar 1988; Willacy \& Millar 1997). This class of models assume an isotropic geometry of the CSE with specified values for the mass-loss rate and expansion velocity. The resulting model CSEs consist of an inner region where assumed parent species in the outflow are broken down, an intermediate region where chemical reactions lead to high abundances of daughter species, and an outer region where the daughter species are destroyed by photodissociation. $\mathrm{SO}_{2}$ in these models has been assigned as a daughter species, although it has since been shown to exist as a possible parent species created in the inner wind (Decin et al. 2010). Cherchneff (2006) studied the role of shocks in $\mathrm{SO}_{2}$ formation in the inner wind within a few stellar radii of the photosphere.

Observational works (e.g., Jackson \& Nguyen 1988; Yamamura et al. 1999; Decin et al. 2010) have repeatedly shown $\mathrm{SO}_{2}$ abundance to be higher both in the extended CSE and the inner wind than expected from CSE and inner wind chemistry models (Willacy \& Millar 1997; Cherchneff 2006). Local abundance of $\mathrm{SO}_{2}$ may be enhanced in the ISM by the passage of shocks (Hartquist et al. 1980), and shock chemistry has been proposed to explain the high observed $\mathrm{SO}_{2}$ relative abundances in CSEs (Jackson \& Nguyen 1988). Alternatively, low model abundances of $\mathrm{SO}_{2}$ presented in Nejad \& Millar (1988) compared to that of Scalo \& Slavsky (1980) may be due to the latter's lower assumed value for the photodissociation of $\mathrm{SO}_{2}$, which is the main mode of destruction of this molecule at large radii (Sahai \& Wannier 1992).

Interferometric mapping of $\mathrm{SO}_{2}$ distribution in the CSE of an evolved star may contribute to the improved understanding of sulfur chemistry by directly constraining the relative abundances of sulfur-bearing species as a function of radius. Furthermore, 
associations of $\mathrm{SO}_{2}$ enhancement with discrete outflow features may favor the idea of localized production and provide evidence for non-isotropic processes in CSEs (Jackson \& Nguyen 1988). In this paper, we present results of high spatial resolution maps obtained from SMA observations of $\mathrm{SO}_{2}$ around VY CMa. We describe four spatially discrete sources of $\mathrm{SO}_{2}$ emission and use multiple transitions of $\mathrm{SO}_{2}$ and derive rotational temperatures and column densities (assuming local thermodynamical equilibrium). We perform non-local thermal equilibrium (LTE) radiative transfer analysis using the RADEX package (van der Tak et al. 2007) to derive local kinetic temperatures, $\mathrm{H}_{2}$ densities, and $\mathrm{SO}_{2}$ abundances of the $\mathrm{SO}_{2}$ emitting regions. We then compare our maps to published visible, IR, and submillimeter observations to evaluate their consistency with the above cited models of circumstellar chemistry.

\section{OBSERVATIONS}

VY CMa was observed with the SMA on 2009 February 18, with the array in extended configuration, offering baselines from 44.2 to $225.9 \mathrm{~m}$. The projected baseline lengths were from 14 to $188 \mathrm{~m}$. The frequency coverage was 234.36 to $236.34 \mathrm{GHz}$ in the lower sideband and 244.36 to $246.34 \mathrm{GHz}$ in the upper sideband. The phase center was at $\alpha(2000)=$ $07^{\mathrm{h}} 22^{\mathrm{m}} 58^{\mathrm{s}} .27, \delta(2000)=-25^{\circ} 46^{\prime} 03^{\prime \prime}$. 4 . The quasars $0730-116$ and $0538-440$ were observed every 20 minutes for gain calibration, and the spectral bandpass was calibrated using quasar $3 c 273$. Flux calibration was done using recent SMA measurements of 0730-116 (2.75 Jy at $1 \mathrm{~mm}$ ) and 0538-440 (3.96 Jy at $1 \mathrm{~mm}$ ). Nominal flux calibration accuracy is $15 \%-20 \%$, depending on the phase stability. In our observations, the uncertainty appears to be better than $10 \%$, based on the good agreement with the ARO spectra (see Section 3.2 and Figure 3). The on-source integration time on VY CMa was $5.66 \mathrm{hr}$. $T_{\text {sys }}(\mathrm{SSB})$ varied approximately from 200 to $400 \mathrm{~K}$ during the track with an atmospheric zenith optical depth of $\sim 0.1$ at the standard reporting frequency of $225 \mathrm{GHz}$, measured at the nearby Caltech Submillimeter Observatory. The conversion factor between Kelvin and Jansky for our observations is $\sim 14 \mathrm{~K} \mathrm{Jy}^{-1}$.

The visibility data were calibrated using the MIR package in IDL and imaged with the MIRIAD software ${ }^{4}$ (Sault et al. 1995). The field of view (FWHM primary beam) varies from $53^{\prime \prime}$ to $56^{\prime \prime}$ whereas the largest angular extent of the source is expected to be about $5^{\prime \prime}$. The synthesized beam size, representing the obtained spatial resolution, is $1^{\prime \prime} .48 \times 1^{\prime \prime}$. 03 . The adopted weighting mode for imaging is "natural" weighting (specified in the Miriad task INVERT). The final rms noise level is $\sim 60 \mathrm{mJy} \mathrm{beam}^{-1}$ per channel in the spectral line images and $3.9 \mathrm{mJy} \mathrm{beam}^{-1}$ in the continuum map. Line emission was subtracted by visually examining the spectra in visibility amplitudes, and using the line-free channels specification to the Miriad task UVLIN.

\section{RESULTS}

\subsection{Continuum Emission}

We detected continuum emission in both the upper and lower sidebands, which is well described by a point source centered at R.A. $=07^{\mathrm{h}} 22^{\mathrm{m}} 58^{\mathrm{s}} 336$, decl. $=-25^{\circ} 46^{\prime} 03^{\prime \prime}$.063. A twodimensional Gaussian fit in the image plane yields an integrated flux of $335.0 \pm 66 \mathrm{mJy}$ at $235.4 \mathrm{GHz}$ and $359.3 \pm 72 \mathrm{mJy}$ at

\footnotetext{
4 http://www.cfa.harvard.edu/ cqi/mircook.html
}

Table 1

Summary of Observed $\mathrm{SO}_{2}$ Lines

\begin{tabular}{lccc}
\hline \hline $\begin{array}{l}\mathrm{SO}_{2} \\
\text { Transition }\end{array}$ & $\begin{array}{c}\text { Frequency } \\
(\mathrm{MHz})\end{array}$ & $\begin{array}{c}E_{u}{ }^{\mathrm{a}} \\
(\mathrm{K})\end{array}$ & $S^{\mathrm{b}}$ \\
\hline $4_{2,2}-3_{1,3}$ & 235151.7 & 19.0 & 1.71 \\
$10_{3,7}-10_{2,8}$ & 245563.4 & 72.7 & 5.44 \\
$15_{2,14}-15_{1,15}$ & 248057.4 & 119.3 & 5.27 \\
$16_{1,15}-15_{2,14}$ & 236216.7 & 130.7 & 6.05 \\
\hline
\end{tabular}

Notes.

${ }^{a} E_{u}$ is the upper rotational state energy.

${ }^{b} S$ is the line strength.

245.4 GHz (maximum errors are of $20 \%$, mainly resulting from the uncertainty in flux calibration). Previous SMA observations of VY CMa at 215 and $225 \mathrm{GHz}$ have yielded continuum fluxes of $270 \pm 40 \mathrm{mJy}$ and $288 \pm 25 \mathrm{mJy}$, respectively (Shinnaga et al. 2004; Muller et al. 2007). These four values are consistent under the Rayleigh-Jeans approximation with blackbody radiation with a brightness temperature higher than $12 \mathrm{~K}$.

\subsection{Line Emission}

In the two sidebands of $\sim 4 \mathrm{GHz}$ bandwidth, we detected a total of 14 lines, which show emissions from $\mathrm{CS}, \mathrm{H}_{2} \mathrm{O}$, $\mathrm{PN}, \mathrm{SiS}$, and $\mathrm{SO}_{2}$. In Figure 1, we present the spectra and maps of the SiS $J=13-12$ and the PN $J=5-4$ lines. These lines are representative of a relatively compact emission that is centered on the peak of the continuum emission, hence they allow us to estimate the systemic velocity of the star. The $\mathrm{SiS}$ spectrum shows the distinctive triple peak morphology as described by Ziurys et al. (2007) and Tenenbaum et al. (2010) that is interpreted as the signature of a slowly expanding shell and a pair of faster outflows nearly collimated with the line of sight. We use the fitted velocity of the central peak of these lines to find the systemic velocity of the star $v_{\mathrm{LSR}}=19.5 \mathrm{~km} \mathrm{~s}^{-1}$. This value is consistent with other results from millimeter and submillimeter observations (Ziurys et al. 2007; Muller et al. 2007) and is adopted as the star's rest-frame velocity in the following sections.

For the remainder of this work we focus only on the $\mathrm{SO}_{2}$ lines, which are summarized in Table 1 . The excitation energies of the four identified $\mathrm{SO}_{2}$ lines vary from 19 to $130 \mathrm{~K}$. All four of these lines were detected in the spectral line survey of Tenenbaum et al. (2010). Our interferometric observations reveal the spatial distribution of the $\mathrm{SO}_{2}$ emission for the first time. The spectra and integrated maps of all four lines are shown in Figure 2. Figure 3 compares the spectra of two $\mathrm{SO}_{2}$ lines with single dish data from the Submillimeter Telescope of the Arizona Radio Observatory, while channel maps of all four lines are presented in Figures 4-7.

Maps of $\mathrm{SO}_{2}$ line maps show well-resolved spatially extended emission over an area of $3^{\prime \prime} \times 5^{\prime \prime}$, in which we distinguish four distinct components, hereafter designated as sources A, B, C, and D (see Table 2). Figure 8 shows all four sources in a position-velocity space diagram of the $245563.4 \mathrm{MHz}$ line. Source positions, orientations, and intensities were fitted to two-dimensional Gaussians using the MIRIAD routine IMFIT. Source $\mathrm{A}$ is a very strong blueshifted source with $v_{\mathrm{LSR}}$ between -18 and $+10 \mathrm{~km} \mathrm{~s}^{-1}$. It is spatially offset to the east of the stellar position by $\sim 0$. 7 . Of similar intensity to source $\mathrm{A}$ is source B, an elongated source offset by $\sim 0$ '. 8 to the west. Source $B$ has a broader spectral profile than source A and is heavily redshifted with $v_{\text {LSR }}$ between +26 and $+66 \mathrm{~km} \mathrm{~s}^{-1}$. Source C 

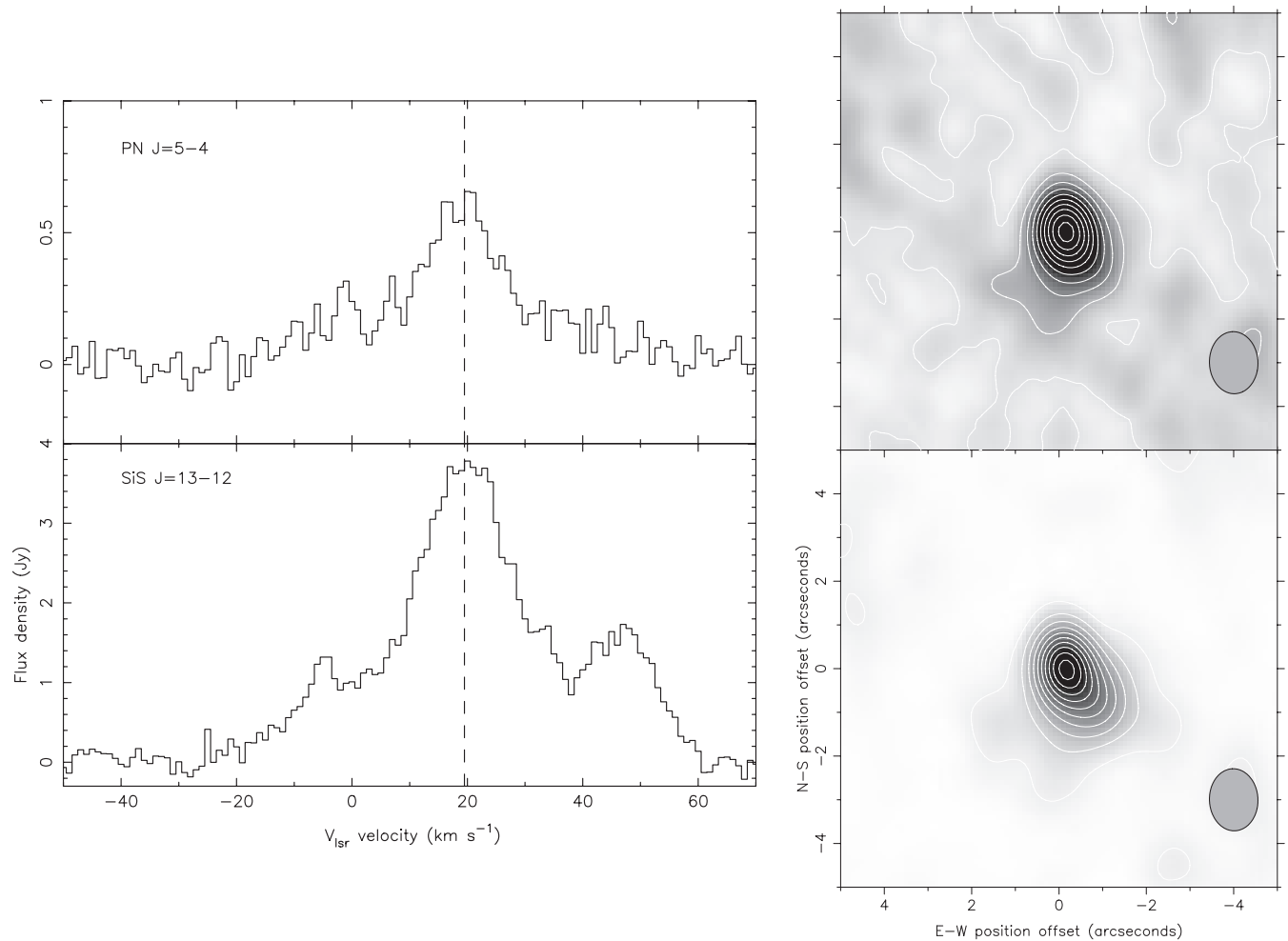

Figure 1. Left: PN $J=5-4$ and SiS $J=13-12$ spectra showing the peak emission at $19.49 \mathrm{~km} \mathrm{~s}^{-1}$ (from the line rest frequencies). We adopt this velocity as that of the stellar frame. Right: integrated intensity maps of PN (top) and SiS (bottom) emission, which show maxima close to the peak of the continuum emission.

Table 2

Description of $\mathrm{SO}_{2}$ Line Emission Sources ${ }^{\mathrm{a}}$

\begin{tabular}{lcccc}
\hline \hline $\begin{array}{l}\text { Source } \\
\left(v_{\text {LSR }} \text { range in } \mathrm{km} \mathrm{s}^{-1}\right)\end{array}$ & $\begin{array}{c}\text { Offset } \\
\left({ }^{\prime \prime}\right)\end{array}$ & $\begin{array}{c}\text { Position } \\
\text { Angle }\left(^{\circ}\right)\end{array}$ & $\begin{array}{c}\text { Size } \\
\left({ }^{\prime \prime}\right)\end{array}$ & Orientation \\
\hline A: $(-18,+10)$ & $0.66 \pm 0.10$ & $33^{\circ}$ & $2.5 \times 2.4$ & $126^{\circ}$ \\
B: $(+26,+66)$ & $0.83 \pm 0.10$ & $316^{\circ}$ & $2.9 \times 1.3$ & $41^{\circ}$ \\
C: $(+26,+66)$ & $2.45 \pm 0.09$ & $272^{\circ}$ & $2.9 \times 1.8$ & $127^{\circ}$ \\
D: $(+12,+26)$ & $\sim 0$ & N/A & $3.2 \times 1.9$ & $\sim-40^{\circ}$ \\
\hline
\end{tabular}

Note. ${ }^{\text {a }}$ Elliptical Gaussian fits of the identified $\mathrm{SO}_{2}$ emission sources. Offsets are measured in arcseconds from the center of the continuum emission (R.A. = $07^{\mathrm{h}} 22^{\mathrm{m}} 58^{\mathrm{s}} 336$, decl. $=-25^{\circ} 46^{\prime} 03^{\prime \prime}$.063). Source size represents the major and minor axis of the ellipse, and its orientation angle is that of the major axis. Angles are given as rotations from north through east. Fits were performed on maps of the $235151.7 \mathrm{MHz}$ line.

is a strong source with a similar radial velocity as source B. In contrast to sources A and B, however, it is heavily offset to the west by $\sim 2$.'5.

Source D is significantly weaker than sources A, B, and C. In velocity space, it is centered on the star's frame of motion $\left(12 \mathrm{~km} \mathrm{~s}^{-1}<v_{\mathrm{LSR}}<26 \mathrm{~km} \mathrm{~s}^{-1}\right)$ with a distinctly narrower spectral profile and similar spatial dimensions as the other sources. It is also spatially centered at the star's location.

Assuming LTE, we can use the integrated intensities measured in the detected $\mathrm{SO}_{2}$ lines in each of the four identified $\mathrm{SO}_{2}$ sources to constrain their respective column density $N$ and rotational temperature $T_{\text {rot }}$, using the expression from Friedel et al. (2005)

$$
\ln \left[\frac{3 c^{2} I_{\text {beam }}}{16 \pi^{3} B \theta_{a} \theta_{b} v^{3} S \mu^{2}}\right]=\ln \left[\frac{N}{Z}\right]-\frac{E_{u}}{T_{\text {rot }}},
$$

where $I_{\text {beam }}$ is the integrated flux in $\mathrm{Jy} \mathrm{km} \mathrm{s}^{-1}$ beam $^{-1}, \theta_{a} \theta_{b}$ is the beam size, and $B$ is the beam filling factor varying from 0.8 to
0.95 for the four distinct sources, as derived from Snyder et al. (2005): $\Theta_{\text {source }} /\left(\Theta_{\text {beam }}+\Theta_{\text {source }}\right) . Z$ is the rotational partition function (Müller et al. 2005), $S$ is the line strength, $\mu$ is the dipole moment (1.63 Debye for $\mathrm{SO}_{2}$ ), $E_{u}$ is the line's upper rotational state energy in $\mathrm{K}$ taken from the JPL spectroscopic database (Pickett 1991), and $\Theta$ is the solid angle.

By performing linear fits of the rotational temperature diagram shown in Figure 9, we find that the three faster outflows (sources A, B, and C) show variations in rotational temperature. Sources A, B, and C have rotational temperature of $61_{-17}^{+39} \mathrm{~K}$ (one $\sigma$ error), $110_{-29}^{+63} \mathrm{~K}$, and $69_{-17}^{+34} \mathrm{~K}$, respectively. The column densities $(N)$ of all three sources are similar $\left(\mathrm{A}: 9.2_{-4.4}^{+7.8} \times 10^{15} \mathrm{~cm}^{-2}\right.$; B: $1.9_{-0.6}^{+0.8} \times 10^{16} \mathrm{~cm}^{-2}$; C: $\left.1.1_{-0.4}^{+0.7} \times 10^{16} \mathrm{~cm}^{-2}\right)$. The rotational temperature of the close-in source $\mathrm{D}$ is much higher at $240_{-52}^{+91}$ $\mathrm{K}$, while its column density is likely lower than that of the others at $7.6 \pm 1.0 \times 10^{15} \mathrm{~cm}^{-2}$.

The derived parameters for source D are uncertain since a significant portion of flux in its velocity range was missed by our interferometric observations due to the lack of short spacing data (our shortest projected baseline $14 \mathrm{~m}$ ). Comparison with single dish data from the Arizona Radio Observatory shows that features as large as $4^{\prime \prime}$ are fully recovered (Figure 3). Features much larger than $4^{\prime \prime}$ may be subject to an underestimation of its flux, although $4^{\prime \prime}$ is a highly conservative estimate. To address this deficiency, we repeated the rotational temperature diagram analysis for source D using a single dish observation made by the Arizona Radio Observatory $10 \mathrm{~m}$ Submillimeter Telescope (Tenenbaum et al. 2010). Assuming a source size of $4^{\prime \prime} \times$ $4^{\prime \prime}$ we derive a lower rotational temperature of $97_{-31}^{+84}$ and a column density of $1.9_{-1.8}^{+2.9} \times 10^{15} \mathrm{~cm}^{-2}$. Because this column density represents an average over our assumed source size, it cannot be directly compared to our derived column densities, which represent the values at the center of each source. This 
Table 3

Summary of LTE and RADEX Modeling Results

\begin{tabular}{lccccc}
\hline \hline Source & $\begin{array}{c}T_{\text {rot }} \\
(\mathrm{K})\end{array}$ & $\begin{array}{c}\mathrm{SO}_{2} \text { Column Density } \\
\left(\mathrm{cm}^{-2}\right)\end{array}$ & $\begin{array}{c}T_{\text {kin }} \\
(\mathrm{K})\end{array}$ & $\begin{array}{c}\mathrm{H}_{2} \text { Density } \\
\left(\mathrm{cm}^{-3}\right)\end{array}$ & $\begin{array}{c}\mathrm{SO}_{2} \text { Abundance } \\
\left(f_{\mathrm{SO}_{2}}\right)\end{array}$ \\
\hline $\mathrm{A}$ & $61_{-17}^{+39}$ & $9.2_{-4.4}^{+7.8} \times 10^{15} \mathrm{~cm}^{-2}$ & 50 & $\gtrsim 2 \times 10^{8}$ & $7 \times 10^{-10}$ \\
$\mathrm{~B}$ & $110_{-29}^{+63}$ & $1.9_{-0.6}^{+0.8} \times 10^{16}$ & $\sim 75$ & $\gtrsim 3 \times 10^{7}$ & $2 \times 10^{-8}$ \\
$\mathrm{C}$ & $69_{-17}^{+34}$ & $1.1_{-0.4}^{+0.7} \times 10^{16}$ & $\sim 100$ & $\gtrsim 3 \times 10^{7}$ & $8 \times 10^{-9}$ \\
$\mathrm{D}$ & $240_{-52}^{+91}$ & $7.6 \pm 1.0 \times 10^{15 \mathrm{a}}$ & $\sim 220$ & $\gtrsim 4 \times 10^{7}$ & $5 \times 10^{-10}$ \\
\hline
\end{tabular}

Note. ${ }^{\text {a }}$ Values for source D suffer from missing extended flux. Using single dish data, average $T_{\text {rot }}=97 \mathrm{~K}$ and $N=1.8 \times 10^{15} \mathrm{~cm}^{-2}$ assuming that the emitter is an uniform $4^{\prime \prime} \times 4^{\prime \prime}$ region.
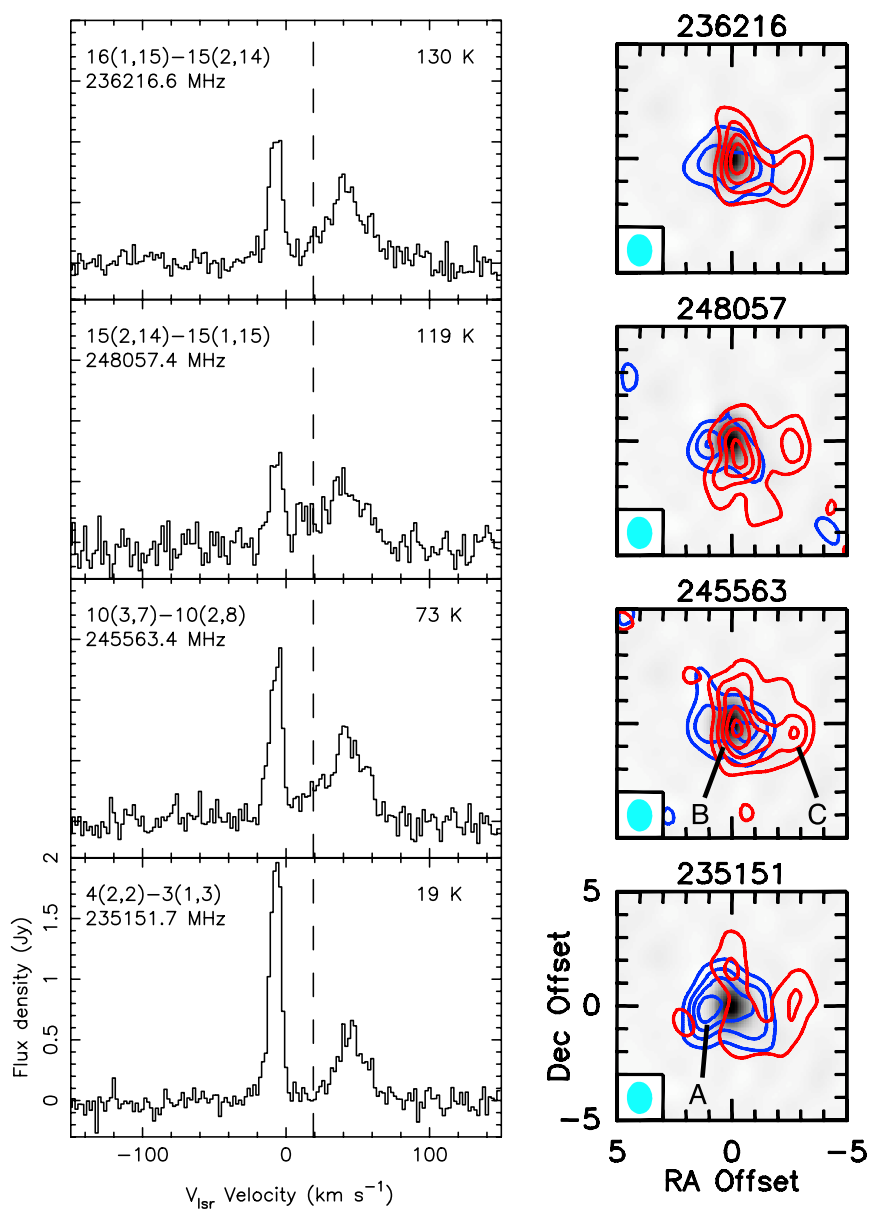

245563
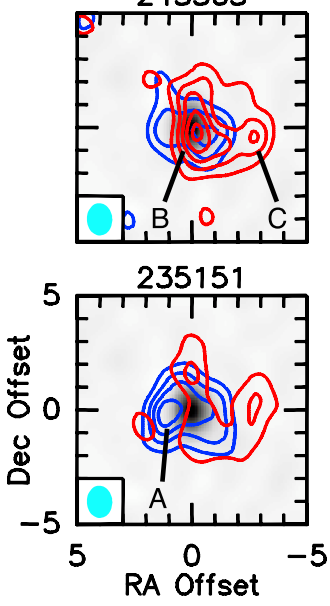

Figure 2. Left: $\mathrm{SO}_{2}$ spectra averaged in a region of $3^{\prime \prime} \times 3^{\prime \prime}$ around the continuum peak. Right: integrated intensity maps corresponding $\mathrm{SO}_{2}$ lines over velocity intervals -20 and $10 \mathrm{~km} \mathrm{~s}^{-1}$ are shown in blue contours. The red contours show the integrated intensity over velocity intervals 20 to $60 \mathrm{~km} \mathrm{~s}^{-1}$. The starting value and interval of contour levels are set to $35 \mathrm{mJy}^{\mathrm{beam}}{ }^{-1} \mathrm{~km} \mathrm{~s}^{-1}$. The continuum emission is shown in gray scale (repeated in all panels). The location of the three identified sources A, B, and C is shown in the bottom two maps.

(A color version of this figure is available in the online journal.)

averaged column density also represents an upper limit due to our assumption of the smallest possible angular size.

The validity of the LTE approximation can be assessed by checking the linearity of the data points in the rotational temperature diagram. Deviation from a linear trend suggests non-LTE conditions in the source or line misidentification. In the case of source $\mathrm{D}$, deviation from linearity is very small compared to the error. We are therefore confident that LTE is a valid assumption for this source. For the outflow sources
A through $\mathrm{C}$, a systematic concave-up shape is noted in the rotation temperature diagram, suggesting departure from LTE conditions.

These findings justify the need to consider a non-LTE radiative transfer model to interpret the measured fluxes. We perform this analysis using the RADEX package (van der Tak et al. 2007), which calculates expected line intensities for a given column density of $\mathrm{SO}_{2}$, kinetic temperature $\left(T_{\text {kin }}\right)$, and volume density of $\mathrm{H}_{2}$. For each source, we assume spatially homogeneous kinetic temperature and volume density. The $\mathrm{SO}_{2}$ column density is fixed for each source and its value is drawn from the LTE analysis, which gives $10^{16} \mathrm{~cm}^{-2}$ for sources A, $\mathrm{B}$, and $\mathrm{C}$ and $10^{15} \mathrm{~cm}^{-2}$ for source D. Varying column density one order of magnitude in each direction does not significantly affect the results. We perform calculations for a wide range of values for the kinetic temperature and the $\mathrm{H}_{2}$ density. We then evaluated the resulting ratios between the flux of each line and that of the $235151.7 \mathrm{MHz}$ transition for the outflow sources A, $\mathrm{B}$, and C. Due to the lack of emission from the $235151.7 \mathrm{MHz}$ line in the vicinity of source D, the $236216.7 \mathrm{MHz}$ line was used instead to calculate line ratios.

Fits to our data are presented as the $\chi^{2}$ statistic $p$-value for each assumed value of $T_{\text {kin }}, \mathrm{H}_{2}$ density (Figure 10), and best-fit results are tabulated in Table 3 . We see that the non-LTE RADEX results for $T_{\text {kin }}$ show general agreement with $T_{\text {rot }}$ derived with LTE assumption. A reliable estimate of $\mathrm{H}_{2}$ density is elusive. Because the flux of each line becomes more similar at greater values of $\mathrm{H}_{2}$ density, we are able only to constrain a lower bound on this value.

The constraints on the temperature and $\mathrm{H}_{2}$ density in source $\mathrm{D}$ are much weaker. However, the best-fit values of $T_{\text {kin }}$ span the $130 \mathrm{~K}$ to $330 \mathrm{~K}$ range, which brackets the value of $T_{\text {rot }}=240 \mathrm{~K}$ obtained from the LTE analysis. This agreement between $T_{\text {rot }}$ and $T_{\text {kin }}$ is consistent with LTE conditions in source D. Our RADEX analysis also allows us to evaluate our assumption of optically thin $\mathrm{SO}_{2}$ lines necessary for the LTE analysis performed above. Although for lower $\mathrm{H}_{2}$ densities $\left(\lesssim 10^{7} \mathrm{~cm}^{-3}\right)$, the $235.151 \mathrm{GHz}$ line comes close to being optically thick, for the inferred $\mathrm{H}_{2}$ densities, all four $\mathrm{SO}_{2}$ have optical thickness below $10^{-2}$.

Assuming that the extent of the sources in the line-of-sight direction is similar to that in the plane of the sky, and that the density is homogenous, we estimate the fractional abundance of $\mathrm{SO}_{2}, f_{\mathrm{SO}_{2}}$ in each source. These are tabulated in Table 3.

\section{DISCUSSION}

\subsection{Source Properties}

We begin by discussing the positions of the four identified $\mathrm{SO}_{2}$ sources. Source C, offset 2 ".5 to the west, is clearly an isolated 

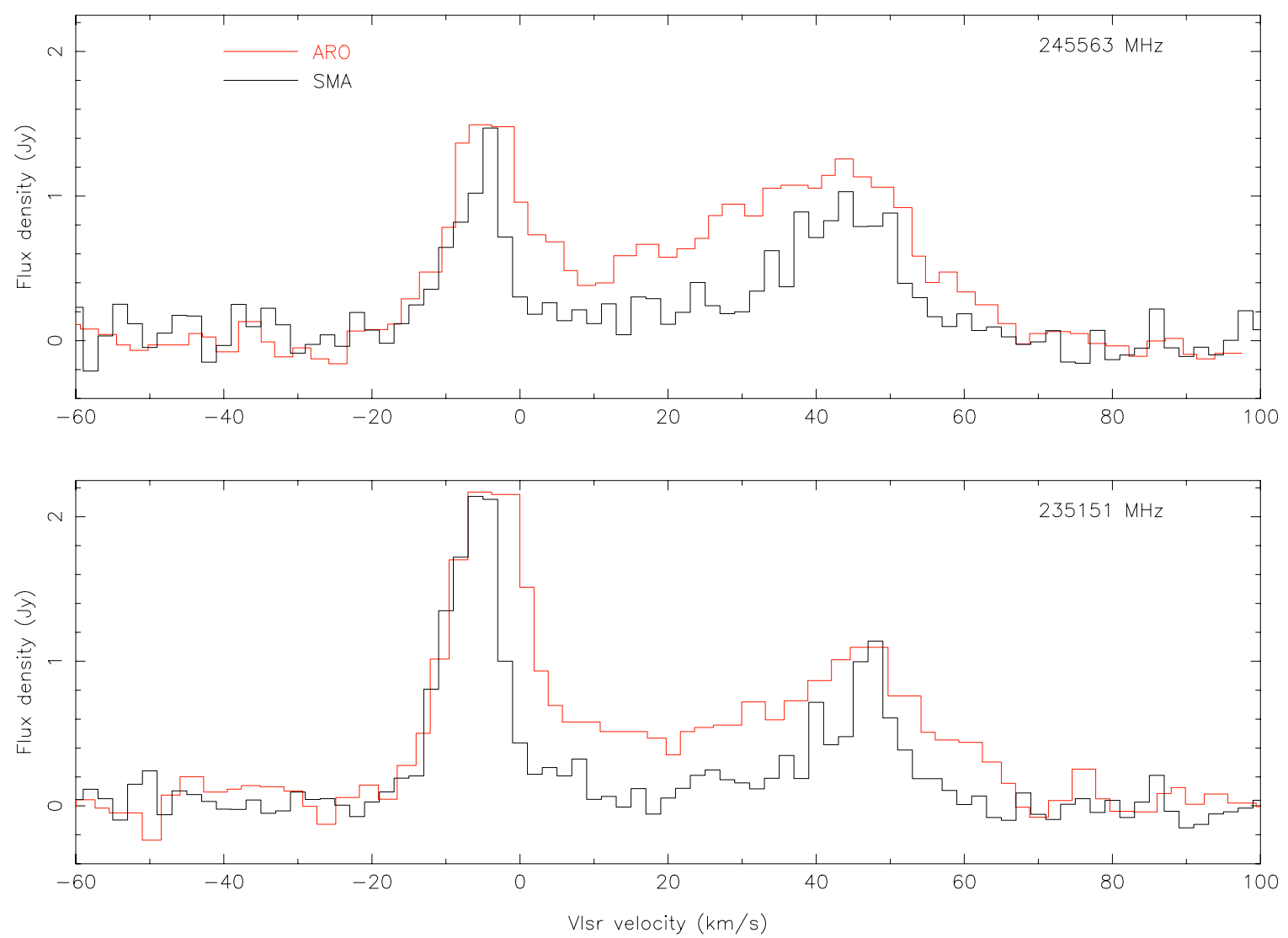

Figure 3. Left: $\mathrm{SO}_{2}$ spectra from our (black) and $10 \mathrm{~m}$ single dish observations by the Arizona Radio Observatory's Submillimeter Telescope (red) for the two lowest temperature transitions (235151.7 and $245563.4 \mathrm{MHz}$ ). The SMA data were smoothed at the appropriate scale to approximate the field of view of the single dish telescope $\left(30^{\prime \prime}\right)$.

(A color version of this figure is available in the online journal.)

body with no antipodal companion to the east. Its existence was suspected in Ziurys et al. (2007), but was not treated as a distinct source. On the other hand, sources A and B are found to be offset in opposite directions relative to the star position with similar blueshift and redshift velocities relative to the stellar frame. SMA observations of the $\mathrm{CO}$ and $\mathrm{SO}$ analogs of sources A and $\mathrm{B}$ were interpreted to be antipodal companions oriented $15^{\circ}$ from the line of sight by Muller et al. (2007). On the other hand, Ziurys et al. (2009) found that single dish observations of CO and other molecules are best explained by a blueshifted and a redshifted source at $20^{\circ}$ and $45^{\circ}$ from the line of sight. Similarly, visible and IR Hubble Space Telescope observations (Humphreys et al. 2007; Smith et al. 2001) have found no evidence of antipodal structure around VY CMa. A careful inspection of sources A and $\mathrm{B}$ in our observation shows that the faster (in radial velocity relative to the star's reference frame) sections of both bodies are offset toward the southwest, while the slower sections are offset toward the northeast. This observation argues against a bipolar geometry, in which antipodal subsections of the two outflows should have similar velocities. We therefore treat sources $\mathrm{A}, \mathrm{B}$, and $\mathrm{C}$ as three distinct outflows probably unrelated to the symmetry axis of the star. These sources do not seem to correspond to any visible or IR features. Our maps show that all $\mathrm{SO}_{2}$ outflow sources are too close to the star to be identified as the "curved nebulous tail" or the numbered arcs presented in Smith et al. (2001), which are located 3".5 from the star.

We identify sources A and B with the blueshifted and redshifted outflows of the previous authors (Ziurys et al. 2007; Muller et al. 2007; Ziurys et al. 2009). The $P-V$ space morphology of these sources match closely with outflows of both CO and SO lines mapped by Muller et al. (2007) (Figure 8). These bodies are hypothesized to have originated in an episode of anomalously high mass loss at uncorrelated locations on the stellar surface (Smith et al. 2001). Assuming that the ages of the outflows are similar and adopting the $\sim 500$ year age found by Muller et al. (2007), we can attempt to derive their locations in three dimensions. We find that the deprojected radii of sources $\mathrm{A}$ and $\mathrm{B}$ are $4.2 \times 10^{16}$ and $4.8 \times 10^{16} \mathrm{~cm}$ and that both are situated at $22^{\circ}$ from the line of sight. Their deprojected starframe velocities of 28 and $30 \mathrm{~km} \mathrm{~s}^{-1}$ fall within the range of measured outflow velocities from the multi-epoch observations of Humphreys et al. (2007). If we assume the same age as for sources $\mathrm{A}$ and $\mathrm{B}$, then source $\mathrm{C}$ is found at a similar radius from the $\operatorname{star}\left(6.9 \times 10^{16} \mathrm{~cm}\right)$, but it is much faster at $44 \mathrm{~km} \mathrm{~s}^{-1}$ and is situated $54^{\circ}$ from the line of sight. $\mathrm{SO}_{2}$ abundance at large radii is expected to be controlled by a balance between the rates of production, expansion, and photodissociation (Scalo \& Slavsky 1980). Outflows with faster expansion velocity are expected to maintain high $\mathrm{SO}_{2}$ abundance out to greater radii, as in the case of our source $\mathrm{C}$.

Source D is elongated and centered at the stellar position, with a resolved minor and major radii of $1^{\prime \prime}$ and $1^{\prime \prime} .6$, corresponding to $2.2 \times 10^{16}$ and $3.5 \times 10^{16} \mathrm{~cm}$. The minor axis radius corresponds to between 180 and 540 stellar radii, depending on the adopted stellar radius (Monnier et al. 1999; Massey et al. 2006). A significant proportion of source D flux is missing from our observation when compared to single dish results (Figure 3). The source we observe therefore appears to represent the warm 

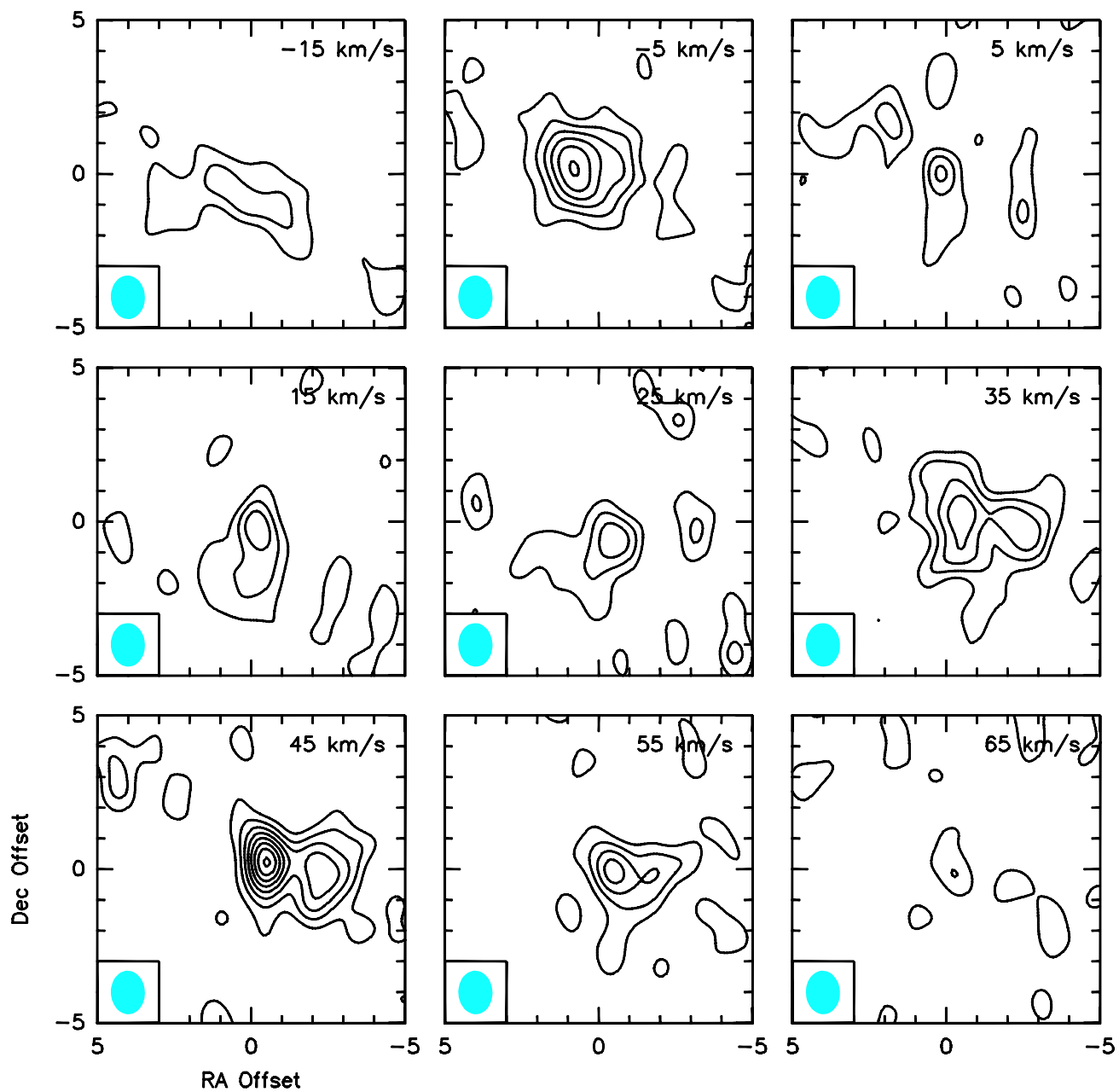

Figure 4. Channel maps of $\mathrm{SO}_{2} 4(2,2)-3(1,3)$ emission at $235151.7 \mathrm{MHz}$ toward VY CMa. In each panel, emission is integrated over a $10 \mathrm{~km} \mathrm{~s}^{-1}$ wide velocity range centered on the velocity indicated on the top right corner. Contour levels are the same as in Figure 2.

(A color version of this figure is available in the online journal.)

core of a larger extended envelope found at the systemic velocity with lower average column density. This extended source is analogous to the spherical wind described in previous millimeter wavelength observations of VY CMa by Ziurys et al. (2007), Ziurys et al. (2009), Muller et al. (2007), and Tenenbaum et al. (2010); however, only the first and last of these works observed $\mathrm{SO}_{2}$ and did not identify it in the spherical wind. These previous works have found a relatively low expansion velocity of between 15 and $20 \mathrm{~km} \mathrm{~s}^{-1}$, which is high given the narrow velocity range of source in our channel maps (Figures 4-7). However, this discrepancy may be due to missing source D flux in our observations. The rotational temperatures derived from SMA and ARO data indicate the existence of a thermalized compact region with elevated temperatures with diameter $\approx 3 \times 10^{16} \mathrm{~cm}$. In the sections below, we refer to this inner region as the core of source D.

Our derived values of $T_{\text {rot }}$ and $T_{\text {kin }}$ distinguish between the lower temperatures of sources $\mathrm{A}, \mathrm{B}$, and $\mathrm{C}$ and a much hotter core of source D. In comparison to previous works, our temperatures for source $\mathrm{A}, \mathrm{B}$, and $\mathrm{C}$ bracket the range of temperatures derived by Muller et al. (2007) and Ziurys et al. (2009) ( $57 \mathrm{~K}$ and $85 \mathrm{~K}$, respectively). This may be expected, as the preceding authors adopted the same best-fit power-law temperature profile for both outflows, which are assumed to be at the same radius. As such, these previous results may represent an average of the temperatures of the outflows.

Our inferred $\mathrm{H}_{2}$ densities from RADEX radiative transfer analysis are higher than the values found in previous studies. Ziurys et al. (2009) adopted an isotropic $\mathrm{H}_{2}$ density profile based on an assumed mass-loss rate that gives a value of $\sim 1 \times 10^{6} \mathrm{~cm}^{-3}$ at $10^{16} \mathrm{~cm}$ radius. Muller et al. (2007) use a similar procedure to arrive at a lower value of $4.5 \times 10^{5} \mathrm{~cm}^{-3}$ at the same location. A higher density of $3 \times 10^{6} \mathrm{~cm}^{-3}$ is assumed for the inner wind region in Tenenbaum et al. (2007).

Part of this discrepancy between our values for $\mathrm{H}_{2}$ density and that of previous authors may be explained by the latter's assumption of isotropic mass flow, which does not account for density concentration in the spatially confined outflow regions. However, this reason alone may not be able to account for the more than two orders of magnitude difference. More significantly, our derived densities of between $5 \times 10^{6}$ and $2 \times 10^{8} \mathrm{~cm}^{-3}$ may be due to the presence of $\mathrm{SO}_{2}$ in regions of local density enhancement above the expected values from an isotropic model. We speculate that such high-density regions are the result of shocks, and their existence around VY CMa can be inferred from the observations of $\mathrm{OH}$ masers, which overlap with sources $\mathrm{A}$ and $\mathrm{B}$ of $\mathrm{SO}_{2}$ emission (Bowers et al. 1983). The activation of the observed $1612 \mathrm{MHz}$ maser line requires $\mathrm{H}_{2}$ densities of between $1 \times 10^{6}$ and $3 \times 10^{7} \mathrm{~cm}^{-3}$ at $100 \mathrm{~K}$ 

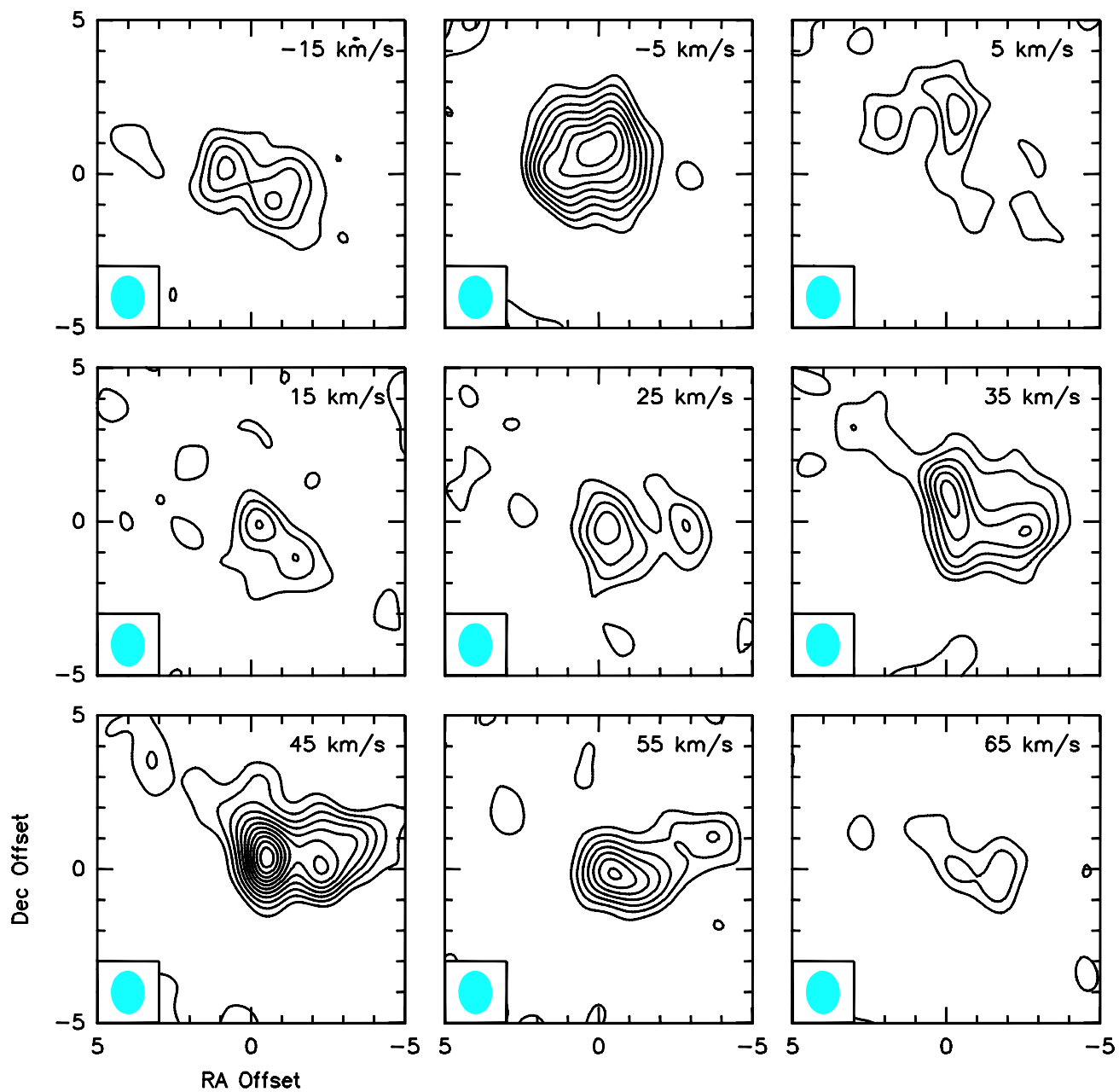

Figure 5. Same as in Figure 4 for $\mathrm{SO}_{2}$ 10(3,7)-10(2,8) emission at $245563.4 \mathrm{MHz}$.

(A color version of this figure is available in the online journal.)

(Pavlakis \& Kylafis 1996). The upper end of this range is similar to our inferred value of minimum $\mathrm{H}_{2}$ density for sources $\mathrm{B}$ and $\mathrm{C}$, while that of source $\mathrm{A}$ is much higher. However, $\mathrm{OH}$ masers may still be active in our source A despite its high density since its temperature of $\sim 55 \mathrm{~K}$ is cooler than that assumed in Pavlakis \& Kylafis (1996).

\subsection{Sulfur Chemistry in the CSE}

For the remainder of the discussion, we address the implications of our observations for circumstellar chemistry by comparing $\mathrm{SO}_{2}$ distribution with those of the $\mathrm{OH}$ and $\mathrm{SO}$ molecules. $\mathrm{SO}_{2}$ in CSEs is formed via the following reaction (Scalo \& Slavsky 1980; Nejad \& Millar 1988; Willacy \& Millar 1997; Cherchneff 2006):

$$
\mathrm{SO}+\mathrm{OH} \longrightarrow \mathrm{SO}_{2}+\mathrm{H} .
$$

The radial abundance of $\mathrm{SO}_{2}$ is therefore expected to reflect that of the two reactant molecules. A striking similarity between the spectral profiles of $\mathrm{SO}_{2}$ and $\mathrm{OH}$ masers has already been noted by Ziurys et al. (2007). Maps of the $1612 \mathrm{MHz} \mathrm{OH}$ maser line show that it coincides with $\mathrm{SO}_{2}$ in sources $\mathrm{A}$ and $\mathrm{B}$, but it is weak or undetectable in the outflow source $\mathrm{C}$ or the spherical wind source $\mathrm{D}$. The lack of $\mathrm{OH}$ maser detection in source $\mathrm{C}$ (or perhaps a very weak detection; see Bowers et al. 1983) may be explained by anisotropic nature of maser radiation. Assuming that the velocity of outflows is oriented radially away from the star, relative velocities between different clumps of gas along a photon's line of travel are smallest when the path is parallel or antiparallel to the gas expansion velocity. The most efficient pumping of a masering state is then achieved when photons travel radially inward or outward from the star. Therefore, the strongest maser emissions are observed from sources along our line of sight (Elitzur et al. 1976). This condition is nearly met for sources $\mathrm{A}$ and $\mathrm{B}$ ( $15^{\circ}$ to $22^{\circ}$ from the line of sight). On the other hand source $\mathrm{C}$, found at a line-of-sight angle of $54^{\circ}$, may also produce the $1612 \mathrm{MHz} \mathrm{OH}$ maser, but its signal is weak along the line of sight.

The weak $\mathrm{OH}$ maser emission in the stellar velocity frame may be attributed to the high kinetic temperature of gas in this region. For a given volume density of gas, temperatures above a certain threshold tend to induce thermal equilibrium in the emitting body, undoing the population inversions responsible for maser emissions (Pavlakis \& Kylafis 1996). For temperatures of $\sim 200 \mathrm{~K}$, the maximum allowable $\mathrm{H}_{2}$ density for the $1612 \mathrm{MHz}$ $\mathrm{OH}$ maser is $3 \times 10^{6} \mathrm{~cm}^{-3}$, which is more than an order of magnitude lower than our inferred density for source D. The highly linear trend of source D data in the rotational temperature diagram (Figure 9) corroborates the prevalence of LTE conditions in source D.

We therefore find that $\mathrm{OH}$ and $\mathrm{SO}_{2}$ distributions are generally similar and that their differences are reconcilable. 

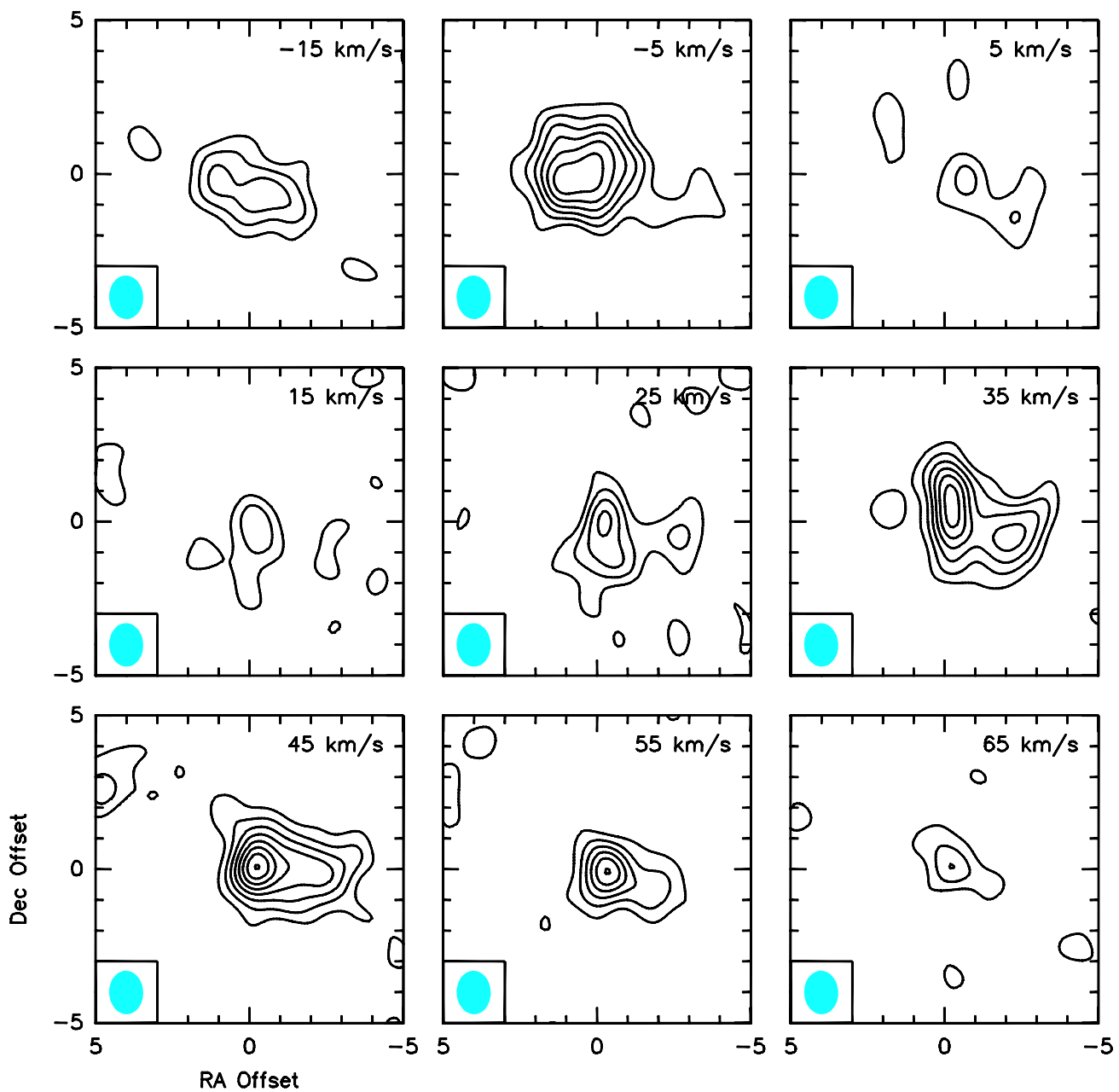

Figure 6. Same as in Figure 4 for $\mathrm{SO}_{2}$ 15(2,14)-15(1,15) emission at 248057.4 MHz.

(A color version of this figure is available in the online journal.)

Finally, we address the discrepancies between the $\mathrm{SO}_{2}$ and SO distributions of Muller et al. (2007), who have mapped the distribution of SO around VY CMa at a similar resolution to ours using a single rotational line of SO $\left(J=6_{5}-5_{4} ; E_{u}=35 \mathrm{~K}\right)$. $\mathrm{SO}$ was found in all four source regions described in this work, although the redshifted $\mathrm{SO}$ emitter is not fragmented into two discrete sources as in the case of $\mathrm{SO}_{2}$. Because of the strongly contrasting values of $\mathrm{H}_{2}$ density adopted in Muller et al. (2007) and this work, direct comparisons between column densities are more instructive than comparisons between fractional abundances. For column density to act as a valid proxy for abundance, we must assume that the line-of-sight dimension of the corresponding $\mathrm{SO}$ and $\mathrm{SO}_{2}$ sources are similar and that the distribution of the each molecules within each source is homogeneous. While we cannot be certain that the line-of-sight thickness of the $\mathrm{SO}$ and $\mathrm{SO}_{2}$ sources are equal, their plane of sky dimensions are similar ( $\sim 30 \%$ discrepancies). The combined column density of both SO outflows and the inner wind where they overlap along the line of sight was found to be $\sim 10^{16} \mathrm{~cm}^{-2}$. This value reflected the combined column density from both outflows and the spherical wind (corresponding to our sources A, $\mathrm{B}$, and D). Given that the three regions contribute approximately equal amounts to the total SO column density, the value of $N_{\text {SO }}$ in each region is on the order of a few $10^{15} \mathrm{~cm}^{-2}$. In contrast, we find column density of $\mathrm{SO}_{2}$ to be 1 to $2 \times 10^{16} \mathrm{~cm}^{-2}$ in each outflow source. The column density of $\mathrm{SO}_{2}$ in the outflow sources $\mathrm{A}$ and $\mathrm{C}$ is therefore $\gtrsim 3$ times greater than that of $\mathrm{SO}$ in the same regions. This statement likely applies as well to outflow source $\mathrm{C}$, given its similar $\mathrm{SO}_{2}$ column density compared to the other outflows and the lack of a discrete $\mathrm{SO}$ source at its location. Furthermore, even with missing flux, the column density of $\mathrm{SO}_{2}$ in the compact core of source D is within error as that of the outflow sources, implying that $N_{\mathrm{SO}_{2}}>N_{\mathrm{SO}}$ in the core of the spherical wind $<3 \times 10^{16} \mathrm{~cm}$ from the star.

Non-equilibrium CSE chemistry models (Scalo \& Slavsky 1980; Nejad \& Millar 1988; Willacy \& Millar 1997) make differing predictions about the ratio of $\mathrm{SO}_{2}$ to $\mathrm{SO}$ abundance $\left(f_{\mathrm{SO}_{2}} / f_{\mathrm{SO}}\right)$. The Scalo \& Slavsky (1980) (SS) model adopts a reaction rate for $\mathrm{SO}_{2}$ formation that is fast compared to the rate of $\mathrm{SO}_{2}$ destruction via photodissociation. Under these conditions, $\mathrm{SO}$ is quickly converted into $\mathrm{SO}_{2}$, which becomes the primary $\mathrm{S}$-bearing species. $\mathrm{SO}_{2}$ therefore reaches maximum abundance at a greater radius than SO. SS has predicted the radius of this transition where $f_{\mathrm{SO}_{2}} / f_{\mathrm{SO}}>1$ to be around several times $10^{15} \mathrm{~cm}$.

Our observations suggest that $f_{\mathrm{SO}_{2}} / f_{\mathrm{SO}}>1$ for the outflow regions $\left(>4 \times 10^{16} \mathrm{~cm}\right.$ from star) and in the compact region within $3 \times 10^{16} \mathrm{~cm}$ of the star. If there does exist a transitional radius at which $f_{\mathrm{SO}_{2}} / f_{\mathrm{SO}}=1$, it must be less than the latter radius, making our study consistent with the SS model. On the other hand, models that predict $f_{\mathrm{SO}_{2}} / f_{\mathrm{SO}}<1$ at all radii are inconsistent with our data (Nejad \& Millar 1988; 

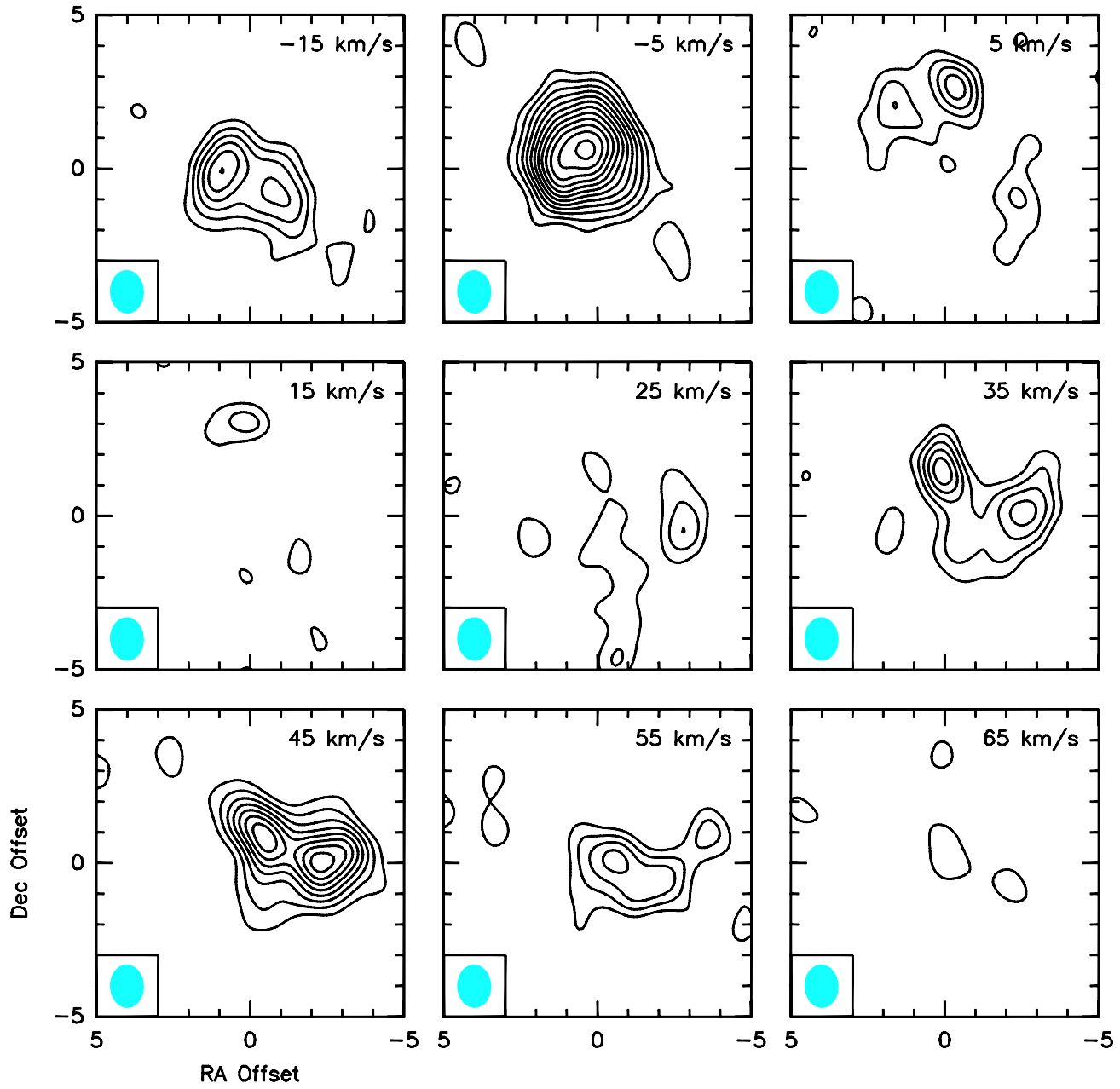

Figure 7. Same as in Figure 4 for $\mathrm{SO}_{2}$ 15(1,15)-15(2,14) emission at $236216.6 \mathrm{MHz}$.

(A color version of this figure is available in the online journal.)

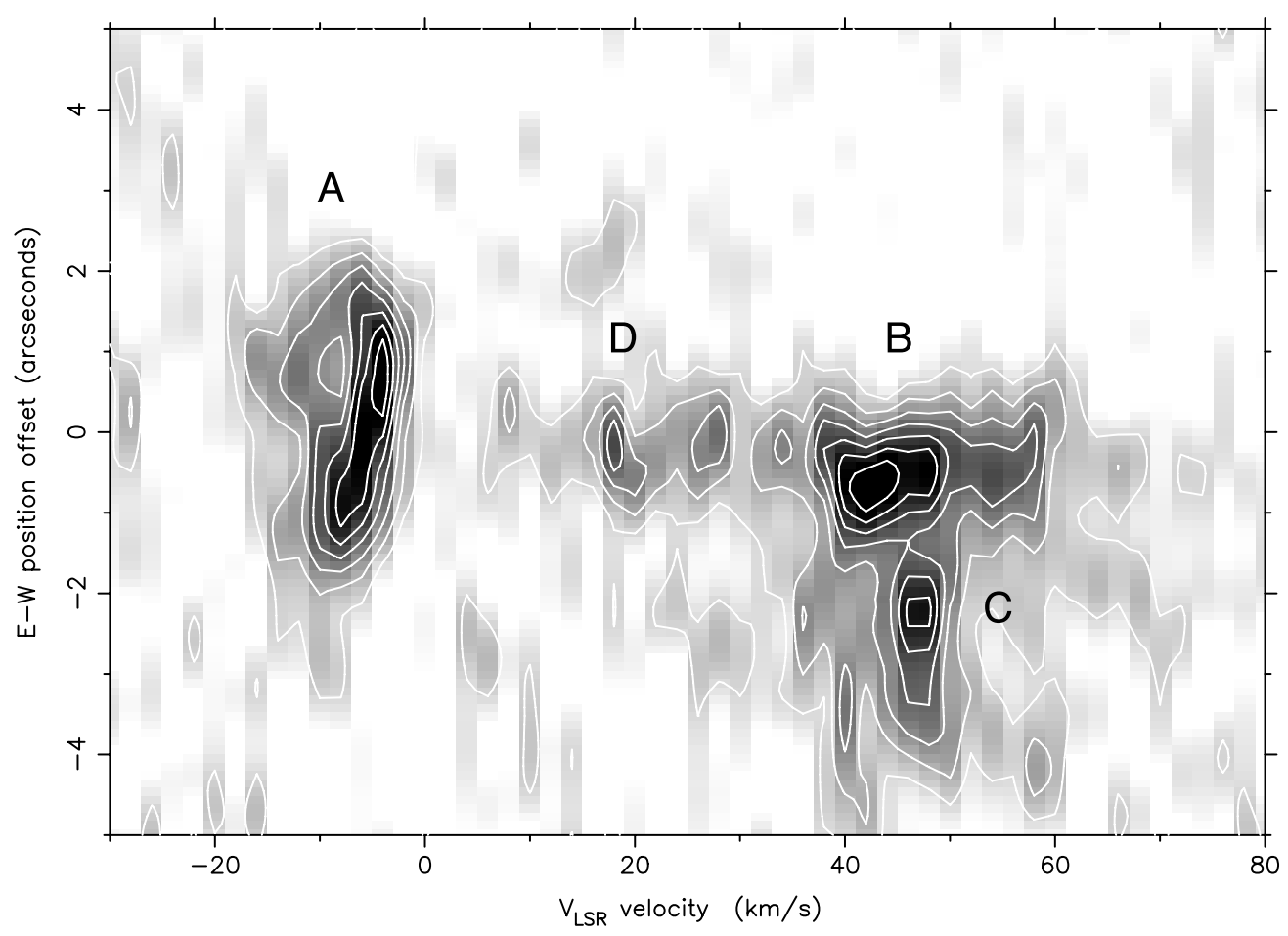

Figure 8. Left: a position velocity cut along east-west at declination offset of $0^{\prime \prime}$ in the $\mathrm{SO}_{2}$ line emission at $245563.4 \mathrm{MHz}$, at position angle $105^{\circ}$. The contours correspond to $10 \%$ of the peak emission. Sources A, B, C, and D are clearly separated. 


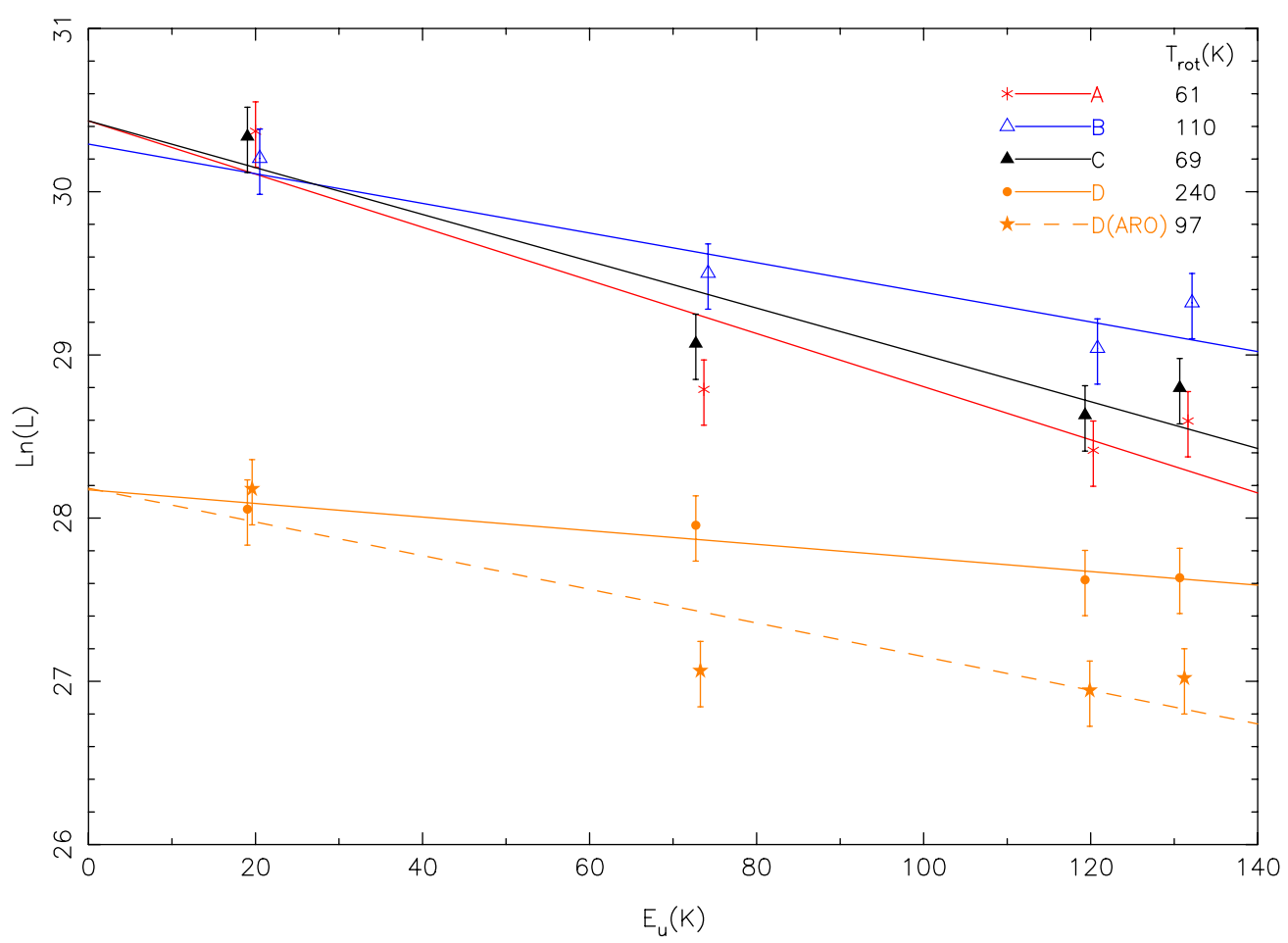

Figure 9. Rotational temperature diagram of the four $\mathrm{SO}_{2}$ emission sources. Red, blue, and black curves and points indicate sources $\mathrm{A}, \mathrm{B}$, and $\mathrm{C}$, respectively. Orange represents the spherical wind (source D). Variable "L" on the vertical axis represents the left-hand side of Equation (1). The dashed orange line represents the spherical wind based on ARO single dish data (Tenenbaum et al. 2010). The errors plotted are set to $3 \sigma$. Horizontal coordinates of the plotted points have been staggered for clarity.

(A color version of this figure is available in the online journal.)

Willacy \& Millar 1997). In addition, the SS model predicts a steady increase in the value of $f_{\mathrm{SO}_{2}} / f_{\mathrm{SO}}$ outward from the $f_{\mathrm{SO}_{2}} / f_{\mathrm{SO}}=1$ transition radius at $\sim 2 \times 10^{15} \mathrm{~cm}$. The value of $f_{\mathrm{SO}_{2}} / f_{\mathrm{SO}}$ increases by more than an order of magnitude by radius $10^{16} \mathrm{~cm}$. Observations of the outflow sources $\mathrm{A}, \mathrm{B}$, and $\mathrm{C}$ do not support this view, as $f_{\mathrm{SO}_{2}} / f_{\mathrm{SO}}$ in on the order of 3 for all four sources. More precise comparisons between $\mathrm{SO}_{2}$ and SO abundance in each source is hindered by the lack of more detailed interpretations of SO maps (the Muller et al. 2007 observation included only one SO line). Therefore, quantitative comparison between model radial distribution of the two species and our results is elusive and we regard our support of the SS model as only qualitative.

Other uncertainties remain in our understanding of sulfur chemistry in the CSE of VY CMa. Our observations cannot be used to constrain whether the $\mathrm{SO}_{2}$ originates in the CSE, as assumed by the cited models, or in shocks in an "inner wind" within a few stellar radii of the photosphere as modeled by Cherchneff (2006) and observed by Decin et al. (2010) and Yamamura et al. (1999). Furthermore, as proposed earlier by Jackson \& Nguyen (1988) and Willacy \& Millar (1997), shock events similar to those that occur in the inner wind may be the source of $\mathrm{SO}_{2}$ enrichment in the outflow lobes. Indeed, comparison of $\mathrm{SO}_{2}$ and maps of $\mathrm{SO}, \mathrm{PN}$, and $\mathrm{SiS}$ (Muller et al. 2007; Figure 1, this work) shows that $\mathrm{SO}_{2}$ exhibits a more fragmented distribution with discrete sources in the redshifted outflow. Unlike these other molecules, the redshifted $\mathrm{SO}_{2}$ outflow is partitioned into two regions of high local abundance (sources B and C), suggesting that local effects may participate in the formation of $\mathrm{SO}_{2}$. However, CSE chemistry models that include the effect of shocks (similar to Cherchneff 2006) remain to be done.
A further uncertainty involves the very high mass loss rate of VY CMa, which is, for example, about two orders of magnitude faster than the asymptotic giant branch star IK Tau (Olofsson et al. 1998). Varying the mass-loss rate in CSE chemistry models does not significantly affect the radial abundance profiles of chemicals species while it does result in globally larger envelopes for higher mass-loss rates (Willacy \& Millar 1997). Therefore, the discussions of the $f_{\mathrm{SO}_{2}} / f_{\mathrm{SO}}$ profile in this work are also relevant to stars with lower mass-loss rates, while the actual measured radii of peak $\mathrm{SO}_{2}$ abundance around VY CMa are unique to this object.

\subsection{Summary}

1. Four rotational lines of the $\mathrm{SO}_{2}$ molecule are mapped with $\sim 1^{\prime \prime}$ resolution around VY CMa. $\mathrm{SO}_{2}$ is found in four discrete sources, three of which are fast (28 to $44 \mathrm{~km} \mathrm{~s}^{-1}$ ) outflows far from the star and one is a slower spherical wind near the star. No symmetrical relationship among the faster outflows or visible and IR features are found.

2. The three fast outflows are found at similar distances from the star and probably originated around 500 years ago.

3. Comparison between our $\mathrm{SO}_{2}$ maps and those of the $1612 \mathrm{MHz} \mathrm{OH}$ maser line suggests that the two species are strongly correlated and that the $\mathrm{OH}$ maser detection may be limited by high temperature and density in the spherical wind.

4. $\mathrm{SO}_{2}$ is more abundant than $\mathrm{SO}$ in all three outflow sources, supporting the non-equilibrium chemistry model of Scalo \& Slavsky (1980). It is inconsistent with models that predict $f_{\mathrm{SO}_{2}} / f_{\mathrm{SO}}<1$ for all radii (e.g., Nejad \& Millar 1988; Willacy \& Millar 1997). The distribution of $\mathrm{SO}_{2}$ in discrete clumps when compared to other molecules may point to the 


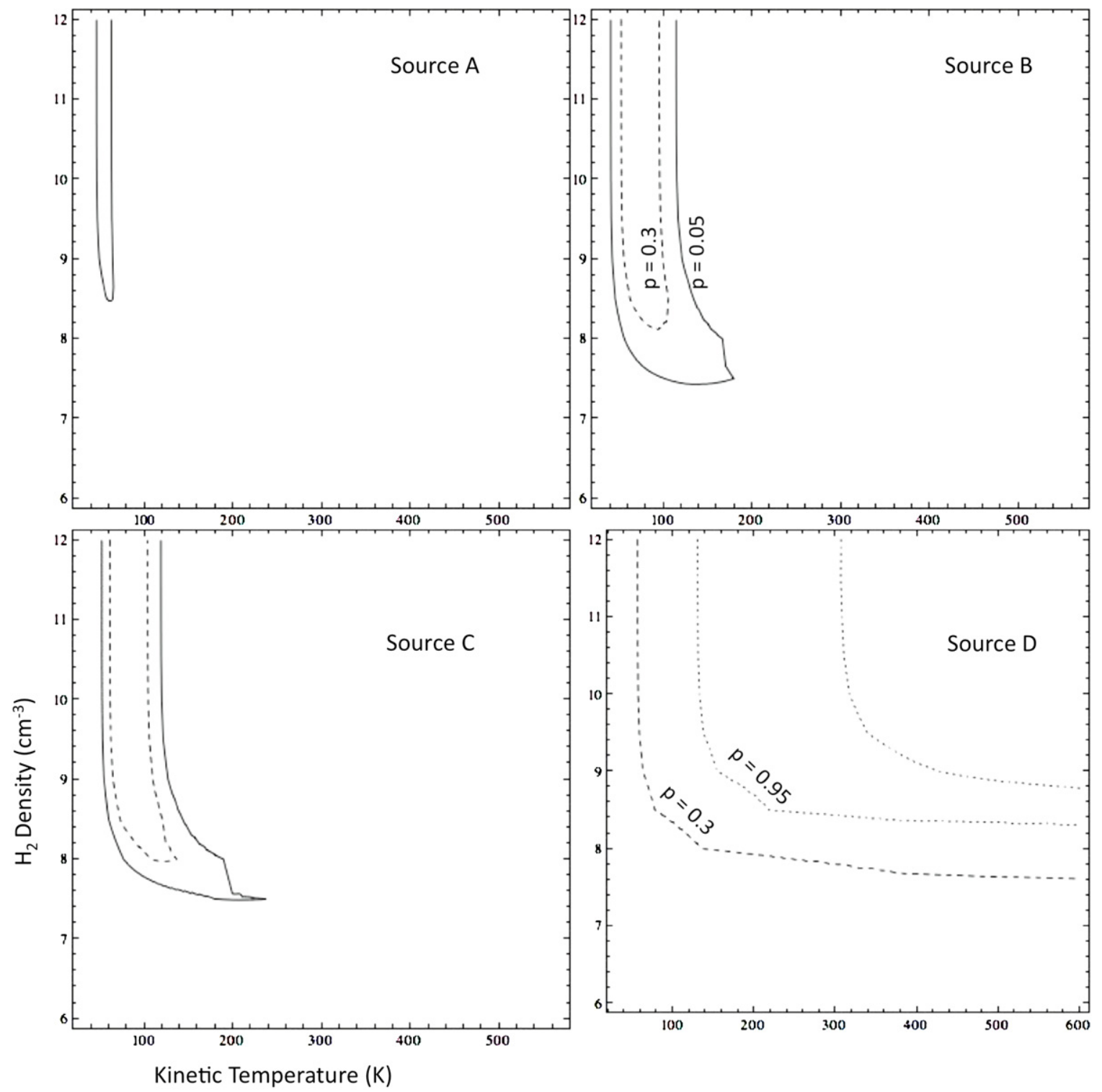

Figure 10. Fits between our measured line ratios and predictions with RADEX radiative transfer. Solid, dashed, and dotted contours enclose regions of $\chi^{2}$ test $p$-values greater than $5 \%, 30 \%$, and $95 \%$. Owing to lower $\mathrm{S} / \mathrm{N}$ and weak dependence of line ratios to these quantities, the derived temperature, and density of source $\mathrm{D}$ are much less well constrained than the other three.

role of localized effects, such as shocks, in the enhancement of $\mathrm{SO}_{2}$ abundance.

We are grateful to Raymond Blundell, Thomas Dame, and Patrick Thaddeus for the opportunity to carry out this research using the SMA as part of the Laboratory Astrophysics (Astro 191) course at Harvard University. We also thank Carl Gottlieb for helpful comments on the manuscript.

\section{REFERENCES}

Bowers, P. F., Johnston, K. J., \& Spencer, J. H. 1983, ApJ, 274, 733 Cherchneff, I. 2006, A\&A, 456, 1001

Danchi, W. C., Bester, M., Degiacomi, C. G., Greenhill, L. J., \& Townes, C. H 1994, AJ, 107, 1469

Decin, L., De Beck, E., Brünken, S., et al. 2010, A\&A, 516, A69

de Wit, W. J., Oudmaijer, R. D., Fujiyoshi, T., et al. 2008, ApJ, 685, L75

Elitzur, M., Goldreich, P., \& Scoville, N. 1976, ApJ, 205, 384

Friedel, D. N., Snyder, L. E., Remijan, A. J., \& Turner, B. E. 2005, ApJ, 632, L95

Guilloteau, S., Lucas, R., Omont, A., \& Nguyen-Q-Rieu 1986, A\&A, 165, L1
Hartquist, T. W., Dalgarno, A., \& Oppenheimer, M. 1980, ApJ, 236 182

Humphreys, R. M., Helton, L. A., \& Jones, T. J. 2007, AJ, 133, 2716

Jackson, J. M., \& Nguyen, Q.-R. 1988, ApJ, 335, L83

Massey, P., Levesque, E. M., \& Plez, B. 2006, ApJ, 646, 1203

Monnier, J. D., Tuthill, P. G., Lopez, B., et al. 1999, ApJ, 512, 351

Müller, H. S. P., Schlöder, F., Stutzki, J., \& Winnewisser, G. 2005, J. Mol. Struct., 742, 215

Muller, S., Dinh-V-Trung, Lim, J., Hirano, N., Muthu, C., \& Kwok, S. 2007, ApJ, 656, 1109

Nejad, L. A. M., \& Millar, T. J. 1988, MNRAS, 230, 79

Olofsson, H., Lindqvist, M., Nyman, L.-A., \& Winnberg, A. 1998, A\&A, 329, 1059

Pavlakis, K. G., \& Kylafis, N. D. 1996, ApJ, 467, 309

Pickett, H. 1991, J. Mol. Spectrosc., 148, 371

Royer, P., Decin, L., Wesson, R., et al. 2010, A\&A, 518, L145

Sahai, R., \& Wannier, P. G. 1992, ApJ, 394, 320

Sault, R. J., Teuben, P., \& Wright, M. C. H. 1995, in ASP Conf. Ser. 77, A Retrospective View of Miriad, Astronomical Data Analysis Software and Systems IV, ed. R. A. Shaw, H. E. Payne, \& J. J. E. Hayes (San Francisco, CA: ASP), 433

Scalo, J. M., \& Slavsky, D. B. 1980, ApJ, 239, L73 
Shinnaga, H., Moran, J. M., Young, K. H., \& Ho, P. T. P. 2004, ApJ, 616, L47

Smith, N., Hinkle, K. H., \& Ryde, N. 2009, AJ, 137, 3558

Smith, N., Humphreys, R. M., Davidson, K., et al. 2001, AJ, 121, 1111

Snyder, L. E., Lovas, F. J., Hollis, J. M., et al. 2005, ApJ, 619, 914

Tenenbaum, E. D., Dodd, J. L., Milam, S. N., Woolf, N. J., \& Ziurys, L. M. 2010, ApJS, 190, 348

Tenenbaum, E. D., Woolf, N. J., \& Ziurys, L. M. 2007, ApJ, 666, L29

van der Tak, F. F. S., Black, J. H., Schöier, F. L., Jansen, D. J., \& van Dishoeck, E. F. 2007, A\&A, 468, 627 van Loon, J. T., Cioni, M.-R. L., Zijlstra, A. A., \& Loup, C. 2005, A\&A, 438 273

Willacy, K., \& Millar, T. J. 1997, A\&A, 324, 237

Yamamura, I., de Jong, T., Onaka, T., Cami, J., \& Waters, L. B. F. M. 1999, A\&A, 341, L9

Yoon, S.-C., \& Cantiello, M. 2010, ApJ, 717, L62

Ziurys, L. M., Milam, S. N., Apponi, A. J., \& Woolf, N. J. 2007, Nature, 447, 1094

Ziurys, L. M., Tenenbaum, E. D., Pulliam, R. L., Woolf, N. J., \& Milam, S. N. 2009, ApJ, 695, 1604 